\section{El impacto de la pandemia de COVID-19 en la salud mental de los trabajadores de la salud en Chile: datos iniciales de The Health Care Workers Study}

\author{
RUBÉN ALVARADO ${ }^{1,9}$, JORGE RAMÍREZ ${ }^{2}$, ÍTALO LANIO ${ }^{3}$, \\ MARGARITA CORTÉS ${ }^{4}$, ANTONIA AGUIRRE ${ }^{5}$, \\ PAULA BEDREGAL ${ }^{6}$, KASIM ALLEL ${ }^{7, a}$, \\ THAMARA TAPIA-MUÑOZ ${ }^{8, b}$, MARÍA SOLEDAD BURRONE $^{9}$, \\ GONZALO CUADRA-MALINARICH ${ }^{10}$, RODRIGO GOYCOLEA ${ }^{11}$, \\ FRANCO MASCAYANO ${ }^{12, a}$, JAIME SAPAG ${ }^{13}$, SARA SCHILLING ${ }^{14}$, \\ GONZALO SOTO ${ }^{1}$, CAROLINA TRAUB ${ }^{13}$, \\ SEBASTIÁN VILLARROEL ${ }^{15}$, SEBASTIÁN ALARCÓN ${ }^{16}$
}

\section{Impact of COVID-19 pandemic on the mental health of healthcare workers}

Background: Healthcare workers' mental health was affected by SARS-CoV-2 pandemic. Aim: To evaluate healthcare workers' mental health and its associated factors during the pandemic in Chile. Material and Methods: An online self-reported questionnaire was designed including the Goldberg Health Questionnaire, the Patient Health Questionnaire, (PHQ-9), and the Columbia-Suicide Severity Rating Scale among other questions. It was sent to 28,038 healthcare workers. Results: The questionnaire was answered by 1,934 participants, with a median age of 38 years ( $74 \%$ women). Seventy five percent were professionals, and $48 \%$ worked at a hospital. Fifty nine percent of respondents had a risk of having a mental health disorder, and $73 \%$ had depressive symptoms. Significant associations were found with sex, workplace, and some of the relevant experiences during the pandemic. Fifty one percent reported the need for mental health support, and 38\% of them received it. Conclusions: There is a high percentage of health workers with symptoms of psychological distress, depression, and suicidal ideas. The gender approach is essential to understand the important differences found. Many health workers who required mental health care did not seek or received it.

(Rev Med Chile 2021; 149: 1205-1214)

Key words: COVID-19; Health Personnel; Mental Health.
${ }_{1}^{1}$ Programa de Salud Mental, Escuela de Salud Pública, Facultad de Medicina, Universidad de Chile. Santiago, Chile. 2Programa de Salud Global, Escuela de Salud Pública, Facultad de Medicina, Universidad de Chile. Santiago, Chile.

${ }^{3}$ Residente Especialidad Médica en Salud Pública, Universidad de Chile. Santiago, Chile ${ }^{4}$ Instituto de Investigación y Postgrado en Salud, Facultad de Ciencias de la Salud, Universidad Central de Chile. Santiago, Chile. ${ }^{5}$ Dirección de Salud, Corporación Municipal de Renca. Santiago, Chile.

${ }^{6}$ Departamento de Salud Pública, Facultad de Medicina, Pontificia Universidad Católica de Chile. Santiago, Chile.

${ }^{7}$ Faculty of Infectious and Tropical Diseases,

London School of Hygiene and Tropical

Medicine. London, UK

${ }^{8}$ Department of Behavioural Science and Health, University College London. London, UK.

IInstituto de Ciencias de la Salud,

Universidad de O'Higgins. Rancagua, Chile. ${ }^{10}$ Colegio Médico de Chile.

${ }^{11}$ Facultad de Ciencias de la Salud,

Universidad Central de Chile. Santiago, Chile. ${ }^{12}$ Mailman School of Public Health, Columbia

University. New York, USA.

${ }^{13}$ Departamentos de Salud Pública y

Medicina Familiar, Escuela de Medicina,

Facultad de Medicina, Pontificia Universidad

Católica de Chile. Santiago, Chile.

${ }^{14}$ Escuela de Medicina, Facultad de Medicina,

Universidad de Chile. Santiago, Chile.

${ }^{15}$ Servicio de Salud del Reloncaví.

${ }^{16}$ Escuela de Salud Pública, Facultad de Medicina, Universidad de Chile. Santiago, Chile.

${ }^{\mathrm{a} P h D}(\mathrm{C})$.

${ }^{b} \mathrm{PhD}$ Student.

Estudio realizado sin financiamiento. Los autores declaran no tener conflictos de interés.

Recibido el 5 de febrero de 2021, aceptado el 5 de julio de 2021

Correspondencia a:

Rubén Alvarado:

Independencia 1027, Independencia,

Santiago.

ralvarado@med.uchile.cl
L a pandemia producida por SARS-CoV-2 (COVID-19) a enero de 2021 ha afectado a más de 83 millones de personas, con más de 1,8 millones de muertes en el mundo ${ }^{1}$. En Chile, se han confirmado 629.176 casos y 16.913 fallecimientos $^{2}$. Esta pandemia tiene consecuencias di- rectas y graves desde el punto de vista infeccioso ${ }^{3,4}$, resultando particularmente agresiva, entre otras causas, por la tensión que imponen a los servicios de salud al representar una demanda asistencial intensa en un tiempo acotado.

Estudios iniciales durante esta pandemia han 
mostrado que la salud mental de la población se ve afectada ${ }^{5}$, por la incertidumbre, cuarentenas, el impacto económico, etc. La evidencia de pandemias pasadas muestran efectos en la salud mental en el largo plazo ${ }^{6}$, En Chile se ha documentado preliminarmente este problema ${ }^{7,8}$.

Dentro de la pandemia, los trabajadores sanitarios tienen mayores condiciones de riesgo para afectar su salud mental: mayor riesgo de contagio, cambios en sus condiciones laborales (sobrecarga de tareas, cambio de funciones, toma de decisiones difíciles, etc.), las medidas de manejo poblacional de la emergencia sanitaria (cuarentenas, restricciones al desplazamiento, limitación de actividades, etc.), etc. Todo esto aumenta sus niveles de estrés, pudiendo afectar negativamente su salud mental durante el periodo epidémico y después de este. La evidencia proveniente de brotes epidémicos previos muestra que los trabajadores sanitarios presentan distrés psicológico, ansiedad, depresión y estrés postraumático ${ }^{9,10}$. Lai et al. ${ }^{11}$, en un estudio transversal llevado a cabo al inicio de esta pandemia en Wuhan (enero-febrero de 2020), reportaron que $71,5 \%$ de médicos y enfermeras presentó distrés psicológico, 34,0\% insomnio, 44,6\% ansiedad y $50,4 \%$ síntomas depresivos. Por lo anterior, es importante estudiar esta situación en Chile.

Respecto a los factores de riesgo, estudios basados en esta pandemia ${ }^{11,12}$ y en otros brotes epidémicos (SARS, MERS y Ébola) ${ }^{13,14}$, reportaron que mujeres, enfermeras y médicos especialistas, en contacto directo con personas contagiadas, tienen mayor riesgo de problemas de salud mental. Otros factores descritos fueron el miedo a enfermar y contagiar a sus familias, así como el estigma y discriminación de la comunidad. Por otro lado, mayor especialización y experiencia previa frente a situaciones de crisis se asoció a menor sintomatología $a^{10,15}$. Esta situación está generando preocupación de parte de los organismos internacionales ${ }^{16}$, junto con el desarrollo de posibles intervenciones ${ }^{17}$.

En muchos países, el contagio en el personal de salud ha avanzado muy rápido en los primeros meses $^{18}$, lo que también ha ocurrido en Chile ${ }^{19}$. Un pequeño estudio realizado en nuestro país (125 profesionales de la salud) mostró altos niveles de síntomas depresivos (65\%), ansiedad (74\%), insomnio $(65 \%)$ y distrés $(57 \%)^{20}$.

La carencia de estudios de mayor envergadura nacional e internacional, que involucre a todos los tipos de trabajadores de la salud, que evalúe los efectos de corto y largo plazo, junto a la necesidad de contribuir al diseño de políticas e intervenciones adecuadas, llevó a desarrollar un proyecto colaborativo, con participación en 30 países de cinco continente: "The HEalth caRe wOrkErs Study" (THCWS) (protocolo aceptado en ClinicalTrials.gov, registro: NCT04352634) que cuenta con el respaldo de OPS/OMS.

\section{Material y Método}

THCWS es un estudio de cohorte, con evaluaciones a través de encuesta de autorreporte al inicio, a los 6 y 12 meses. Aquí se describen los datos de la evaluación inicial, como un estudio transversal.

Para la estimación del tamaño muestral mínimo, se tomó como referencia el mencionado estudio de Lai et al. ${ }^{11}$. Se asumió la condición más exigente para el tamaño muestral, con un $\mathrm{p}=50 \%$. Así, con un nivel de confianza de $95 \%$ para hipótesis bilateral y tamaño del error de \pm 3\%, se necesitaban 1.067 participantes a seguir. Estimando una pérdida anual no mayor a $25 \%$, la cohorte debería comenzar con 1.423 personas.

Se diseñó un cuestionario estructurado cuyos contenidos se encuentran descritos en la Tabla $1^{21-26}$.

Durante abril de 2020, se identificaron diferentes tipos de establecimientos de salud interesados en participar en el estudio, y que tuvieran cuatro características: a) que pertenezcan al sector público y al privado; 2 ) que brinden servicios a todos los grupos de edad; 3 ) que incluya todos los niveles de complejidad, desde centros de atención primaria hasta hospitales de alta complejidad; y 4) que haya diversidad regional. Se contactó mediante autoridades a 48 establecimientos y finalmente se contó con 36 de estos, donde se realizó la invitación directa a los trabajadores (a sus correos electrónicos o por mensajes de WhatsApp). Entre el 22 de mayo y el 2 de julio del 2020, se enviaron 28.038 invitaciones, obteniéndose 973 respuestas, 188 de ellas incompletas. Esto equivale a una tasa de participación de 3,4\%. Para incrementar la tasa de participación, se usó un enlace que se distribuyó por diferentes vías (evento de difusión, redes sociales, envío masivo de correos, etc.). Entre el 3 de julio y el 31 de agosto de 2020 se obtuvieron 
Tabla 1. Contenidos de la encuesta aplicada a trabajadores de la salud entre mayo y julio de 2020, Chile

\begin{tabular}{|c|c|}
\hline Contenido & Instrumento \\
\hline $\begin{array}{l}\text { Aspectos socio-demográficos, laborales y antecedentes } \\
\text { de salud }\end{array}$ & Confeccionado ad-hoc \\
\hline Experiencias relevantes durante la pandemia & $\begin{array}{l}\text { Confeccionado ad-hoc para incluir: exposición a pacientes } \\
\text { COVID-19, uso y evaluación de equipos de protección per- } \\
\text { sonal (EPP), contagio y muerte de seres queridos, temores al } \\
\text { contagio, etc. }\end{array}$ \\
\hline Probabilidad de trastornos de salud mental & $\begin{array}{l}\text { Goldberg Health Questionnaire, en su versión de } 12 \text { preguntas } \\
(\text { GHQ-12)* }\end{array}$ \\
\hline Probabilidad de trastorno depresivo & $\begin{array}{l}\text { Patient Health Questionnaire, en su versión de } 9 \text { preguntas } \\
(\mathrm{PHQ}-9)^{\dagger}\end{array}$ \\
\hline Ideación e intento suicida & Escala de Seriedad Suicida de Columbia ${ }^{\ddagger}$ \\
\hline $\begin{array}{l}\text { Autoevaluación del estado de salud mental, y necesidad, } \\
\text { búsqueda y acceso a atenciones de ayuda psicológica }\end{array}$ & Confeccionado ad-hoc \\
\hline
\end{tabular}

*Es uno de los instrumentos más utilizados en el mundo y con buenas propiedades psicométricas para valorar el nivel de malestar psíquico e identificar un posible caso con un trastorno mental ${ }^{18}$. En Chile, existe un estudio de validación de criterio, en el cual se obtuvo que el mejor punto de corte estaba entre 4 y 5 puntos ${ }^{19}$ y se ha demostrado que la versión de 12 preguntas tiene una estructura unifactorial, siendo útil para identificar niveles de distrés o malestar psicológico ${ }^{20}$. ${ }^{\dagger}$ Este instrumento fue creado para la identificación de casos con un trastorno depresivo entre consultante de Atención Primaria y la intensidad de su cuadro ${ }^{21}$, pero que se ha extendido su uso a población general, en especial para identificar síntomas depresivos. Ha sido validado en Chile, mostrando buenas propiedades psicométricas y una estructura unifactorial22. ${ }^{2}$ Preguntas seleccionadas tomadas de esta escala ${ }^{23}$

1.481 respuestas más, 332 de ellas incompletas. De este modo, se recibió un total de 2.454 respuestas, siendo 1.934 completas, que es la muestra final para el análisis de la línea basal del estudio.

Se utilizó una plataforma digital alojada en la Universidad de Chile, que tiene una funcionalidad de manejo y protección de datos semejante a REDCap ${ }^{27}$, y que cuenta con tecnología de encriptación de datos de estándar internacional. Para garantizar la confidencialidad, se utilizó un sistema de códigos "ID" para posterior seguimiento. La seguridad fue garantizada mediante tres elementos: a) ingresos restringidos al sistema solo para el personal con credenciales de acceso asignadas por el administrador; b) datos respaldados diariamente en servidores virtuales que garantizan la seguridad, integridad y disponibilidad de la información; y, c) trazabilidad de cualquier modificación de la base de datos. Para garantizar la calidad de los datos, todas las secciones de la encuesta siguieron un formato digital que cuenta con saltos lógicos y rangos predeterminados (compatible con smartphone, tablet y PC). Esto permite completar correctamente el instrumento e impide la introducción de valores fuera de rango, reduciendo errores de digitación.

El estudio fue aprobado por el Comité de Ética de Investigación de Seres Humanos de la Facultad de Medicina de la Universidad de Chile (protocolo No 024-2020), y por el Ethics Review Committee de la Organización Panamericana de la Salud (PAHOERC.0208.02). Los participantes aprobaron un consentimiento informado antes de iniciar la encuesta.

\section{Resultados}

La Tabla 2 presenta los datos generales de la muestra de 1.934 trabajadores de la salud. Cerca de tres cuartos eran mujeres y la edad promedio fue de 37,7 años. El 41,3\% eran médicos, 33,4\% otros profesionales, $9,1 \%$ técnicos, y $10,1 \%$ trabajaban en gestión o áreas administrativas. La mayoría $(85,3 \%)$ tenía un nivel de educación universitaria o superior. Respecto del lugar de trabajo, 48,2\% laboraba en un hospital y 44,6\% lo hacía en un centro de atención ambulatoria. 
Tabla 2. Características sociodemográficas y laborales de los trabajadores de salud que participaron en el estudio $(n=1.934)$

\begin{tabular}{|c|c|c|}
\hline Variables & Frecuencia & Porcentaje \\
\hline \multicolumn{3}{|l|}{ Género: } \\
\hline - Mujer & 1.432 & $74,1 \%$ \\
\hline - Hombre & 502 & $25,9 \%$ \\
\hline \multicolumn{3}{|l|}{ Nivel educacional: } \\
\hline - Enseñanza Básica / Media & 46 & $2,4 \%$ \\
\hline - Técnico-Profesional & 238 & $12,3 \%$ \\
\hline - Universitaria & 979 & $50,6 \%$ \\
\hline - Postgrado / Especialidad & 671 & $34,7 \%$ \\
\hline \multicolumn{3}{|l|}{ Tipo de trabajo: } \\
\hline - Médicos & 799 & $41,3 \%$ \\
\hline - Otros profesionales & 646 & $33,4 \%$ \\
\hline - Técnicos & 176 & $9,1 \%$ \\
\hline - Administrativos & 110 & $5,7 \%$ \\
\hline - Gestión & 85 & $4,4 \%$ \\
\hline - Otros & 118 & $6,1 \%$ \\
\hline \multicolumn{3}{|l|}{ Tipo de Centro: } \\
\hline - Hospitales & 932 & $48,2 \%$ \\
\hline - Centros de Atención Ambulatoria (CESFAM, CDT, CRS, COSAM, etc.) & 863 & $44,6 \%$ \\
\hline - Otros (Servicios de Salud, SEREMI, etc.) & 139 & $7,2 \%$ \\
\hline Edad (promedio \pm D.E.): & $37,7 \pm 10,3$ años & \\
\hline
\end{tabular}

Nota: D.E. = desviación estándar.

Los centros de atención ambulatoria tuvieron un porcentaje significativamente mayor de mujeres $(79,6 \%)$, respecto de los hospitales $(70,0 \%)$ y las otras dependencias $(67,4 \%)(p<0,001)$. De igual forma, la edad promedio fue significativamente menor en los centros de atención ambulatoria (36,8 $\pm 9,6$ años), respecto del observado en los trabajadores de hospitales $(38,7 \pm 11,0$ años $)$ y de otras dependencias $(37,6 \pm 10,0$ años $)(p<0,001)$.

\section{Experiencias relevantes al trabajar en la pandemia de COVID-19}

Las frecuencias de diferentes experiencias que estuvieron relacionadas con el trabajo en pandemia y que han sido asociadas al estado de salud mental se presentan desagregadas por género (Tabla 3 ) y por lugar de trabajo (Tabla 4).

Las experiencias que resultaron más frecuentes o relevantes fueron:

- Una mayoría señaló haber estado cerca de pacientes con sospecha o confirmación de COVID-19 en la semana previa.
- Sobre la mitad consideró que los equipos de protección personal (EPPs) son suficientes para prevenir el contagio.

- Más de dos tercios mencionó alta preocupación por contagiarse de COVID-19. Esta preocupación fue aun mayor frente al contagio de seres queridos con quienes vive.

- Menos de un tercio refirió que algún familiar había sido diagnosticado de COVID-19 y cerca de $12 \%$ tenía algún familiar que había fallecido por esta enfermedad.

- Más de un tercio refirió haberse sentido estigmatizado o discriminado y cerca de $15 \%$ refirió haber sufrido algún acto de violencia, por su condición de trabajador sanitario.

- Gran mayoría refirió sentir apoyo desde sus compañeros de trabajo y seres queridos.

La Tabla 3 muestra que, para la mayoría de las experiencias señaladas, las trabajadoras eran quienes tienen una situación de mayor riesgo para su salud mental, respecto de los hombres. Una mayor 
proporción cree: que las EPPs son insuficientes, tiene miedo de contagiarse o de contagiar a seres queridos, se ha sentido estigmatizada o discriminada, o ha sido objeto de violencia. Además, un porcentaje menor siente contar con una red fiable en su trabajo.

La Tabla 4 presenta la comparación entre los tres lugares de trabajo, observándose una mayor frecuencia de condiciones de riesgo entre los trabajadores de centros de atención ambulatoria (la mayoría de ellos trabaja en atención primaria).

\section{Frecuencia del malestar psicológico (GHQ-12)}

El puntaje total del GHQ-12 21 para todo el grupo tuvo un promedio de 16,3 y DE 6,3 puntos; un rango entre 1 y 36 puntos, y una distribución que asemeja a una curva normal, pero asimétrica hacia la derecha (asimetría: 0,376 ) y achatada en la punta (curtosis: -0,349). Al comparar por género (Tabla 5), las mujeres tuvieron un puntaje significativamente más elevado que los hombres, $\mathrm{y}$ al hacerlo por lugar de trabajo no se aprecian diferencias significativas.

Al usarlo como tamizaje para casos de trastorno mental ${ }^{22}$, se encontró que $58,9 \%$ de los encuestados eran positivos (puntaje de 5 o más puntos). Las mujeres encuestadas tenían una frecuencia significativamente más elevada de casos positivos $(61,9 \%)$ respecto de los hombres $(50,3 \%)$ y no hay diferencias significativas según lugar de trabajo $(\mathrm{p}=0,582)$.

\section{Frecuencia de sintomas y cuadros depresivos (PHQ-9)}

Basado en el puntaje del PHQ-9 se puede clasificar a los encuestados según intensidad de los síntomas depresivos ${ }^{24,25}$ (Tabla 5): 31,3\% presentaba síntomas depresivos moderados a graves y 5,6\% tenía síntomas graves (36,9\% la suma de ambos grupos).

Tabla 3. Frecuencia de experiencias relevantes del trabajo en la pandemia COVID-19, entre los trabajadores de salud que participaron en el estudio, por sexo y expresado en porcentaje $(n=1.934)$

\begin{tabular}{|c|c|c|c|c|}
\hline \multirow[t]{2}{*}{ Variables } & \multirow{2}{*}{$\begin{array}{l}\text { Toda la } \\
\text { muestra }\end{array}$} & \multicolumn{2}{|c|}{ Género } & \multirow{2}{*}{$\begin{array}{l}\text { Diferencia } \\
\text { de medias } \\
\text { p-value }\end{array}$} \\
\hline & & Mujer & Hombre & \\
\hline \multicolumn{5}{|l|}{$\begin{array}{l}\text { En la última semana, ha estado cerca de pacientes con sospecha o } \\
\text { confirmación de COVID-19 }\end{array}$} \\
\hline • "Si" & $58,2 \%$ & $55,2 \%$ & $67,1 \%$ & $<0,001$ \\
\hline - "No sabe" & $15,0 \%$ & $15,9 \%$ & $12,4 \%$ & \\
\hline $\begin{array}{l}\text { ¿Considera que los equipos (EPPs) que posee son suficientes para evitar } \\
\left.\text { el contagio? ( } \mathrm{Si}^{\prime \prime}\right)\end{array}$ & $55,4 \%$ & $52,7 \%$ & $63,1 \%$ & $<0,001$ \\
\hline ¿Cuánto le preocupa contagiarse? ("Mucho" o "muchísimo") & $70,6 \%$ & $73,1 \%$ & $63,5 \%$ & $<0,001$ \\
\hline $\begin{array}{l}\text { ¿Cuánto le preocupa contagiar a sus seres queridos? ("Mucho" o } \\
\text { "muchísimo") }\end{array}$ & $92,6 \%$ & $93,3 \%$ & $90,6 \%$ & 0,047 \\
\hline $\begin{array}{l}\text { Desde el inicio de la pandemia, ¿algún ser querido ha tenido COVID-19? } \\
(\text { ("Si") }\end{array}$ & $28,8 \%$ & $29,2 \%$ & $27,5 \%$ & 0,484 \\
\hline ¿Algún ser querido ha fallecido por COVID-19? ("Si") & $12,4 \%$ & $11,5 \%$ & $15,2 \%$ & 0,245 \\
\hline $\begin{array}{l}\text { Como trabajador/a de la salud, me he sentido estigmatizado/a y/o } \\
\text { discriminado/a por razones relativas a COVID-19 ("De acuerdo" y "muy } \\
\text { de acuerdo") }\end{array}$ & $39,4 \%$ & $42,4 \%$ & $31,1 \%$ & $<0,001$ \\
\hline $\begin{array}{l}\text { He sufrido violencia por ser trabajador/a de la salud durante la pandemia } \\
\text { ("De acuerdo" y "muy de acuerdo") }\end{array}$ & $15,4 \%$ & $15,8 \%$ & $14,2 \%$ & 0,009 \\
\hline $\begin{array}{l}\text { Cuento con una red fiable de compañeros/as de trabajo ("De acuerdo" } \\
\text { y "muy de acuerdo") }\end{array}$ & $81,7 \%$ & $80,4 \%$ & $85,4 \%$ & 0,046 \\
\hline $\begin{array}{l}\text { Tengo seres queridos que me apoyan cuando lo necesito ("De acuerdo" } \\
\text { y "muy de acuerdo") }\end{array}$ & $93,8 \%$ & $93,1 \%$ & $95,6 \%$ & 0,165 \\
\hline
\end{tabular}

Nota: $\mathrm{EPP}=$ Elementos de protección personal. 
Tabla 4. Frecuencia de experiencias relevantes del trabajo en la pandemia COVID-19, entre los trabajadores de salud que participaron en el estudio, según lugar de trabajo, expresado en porcentaje $(n=1.934)$

\begin{tabular}{|c|c|c|c|c|c|}
\hline \multirow[t]{2}{*}{ Variables } & \multirow{2}{*}{$\begin{array}{l}\text { Toda la } \\
\text { muestra }\end{array}$} & \multicolumn{3}{|c|}{ Tipo de Centro* } & \multirow{2}{*}{$\begin{array}{l}\text { Diferencia } \\
\text { de medias } \\
\text { p-value }\end{array}$} \\
\hline & & Hosp & CAA & Otro & \\
\hline \multicolumn{6}{|l|}{$\begin{array}{l}\text { En la última semana, ha estado cerca de pacientes con } \\
\text { sospecha o confirmación de COVID-19 }\end{array}$} \\
\hline • "Si" & $58,2 \%$ & $65,3 \%$ & $53,7 \%$ & $38,9 \%$ & $<0,001$ \\
\hline • "No sabe" & $15,0 \%$ & $12,0 \%$ & $17,3 \%$ & $21,6 \%$ & \\
\hline $\begin{array}{l}\text { ¿Considera que los equipos (EPPs) que posee son sufi- } \\
\text { cientes para evitar el contagio? ("Si") }\end{array}$ & $55,4 \%$ & $57,1 \%$ & $53,4 \%$ & $56,1 \%$ & 0,347 \\
\hline $\begin{array}{l}\text { ¿Cuánto le preocupa contagiarse? ("Mucho" o "muchí- } \\
\text { simo") }\end{array}$ & $70,6 \%$ & $67,5 \%$ & $73,7 \%$ & $71,7 \%$ & 0,033 \\
\hline $\begin{array}{l}\text { ¿Cuánto le preocupa contagiar a sus seres queridos? } \\
\text { ("Mucho" o "muchísimo") }\end{array}$ & $92,6 \%$ & $91,6 \%$ & $94,2 \%$ & $88,5 \%$ & 0,024 \\
\hline $\begin{array}{l}\text { Desde el inicio de la pandemia, ¿algún ser querido ha } \\
\text { tenido COVID-19? ("Si") }\end{array}$ & $28,8 \%$ & $26,0 \%$ & $31,2 \%$ & $33,1 \%$ & 0,026 \\
\hline ¿Algún ser querido ha fallecido por COVID-19? ("Si") & $12,4 \%$ & $9,9 \%$ & $15,9 \%$ & $4,4 \%$ & 0,027 \\
\hline $\begin{array}{l}\text { Como trabajador/a de la salud, me he sentido estig- } \\
\text { matizado/a y/o discriminado/a por razones relativas a } \\
\text { COVID-19 ("De acuerdo" y "muy de acuerdo") }\end{array}$ & $39,4 \%$ & $40,3 \%$ & $38,2 \%$ & $41,0 \%$ & 0,348 \\
\hline $\begin{array}{l}\text { He sufrido violencia por ser trabajador/a de la salud du- } \\
\text { rante la pandemia ("De acuerdo" y "muy de acuerdo") }\end{array}$ & $15,4 \%$ & $11,4 \%$ & $19,1 \%$ & $18,7 \%$ & $<0,001$ \\
\hline $\begin{array}{l}\text { Cuento con una red fiable de compañeros/as de trabajo } \\
\text { ("De acuerdo" y "muy de acuerdo") }\end{array}$ & $81,7 \%$ & $82,9 \%$ & $80,6 \%$ & $79,9 \%$ & 0,002 \\
\hline $\begin{array}{l}\text { Tengo seres queridos que me apoyan cuando lo necesito } \\
\text { ("De acuerdo" y "muy de acuerdo") }\end{array}$ & $93,8 \%$ & $94,2 \%$ & $93,4 \%$ & $92,8 \%$ & 0,272 \\
\hline
\end{tabular}

Nota: EPP $=$ Elementos de protección personal. Hosp = Hospitales; CAA = Centros de atención ambulatoria; Otro= Otros.

En la Tabla 5 se puede apreciar que entre las mujeres el grupo con sintomatología moderada a grave fue $41,1 \%$, siendo significativamente más alto que en los hombres. No hay diferencias significativas según lugar de trabajo.

\section{Frecuencia de ideas suicidas}

El 12,2\% de los encuestados señaló tener el deseo de estar muerto/a ${ }^{26}$. Entre las mujeres, esta cifra fue notoriamente más elevada $(13,6 \%)$ respecto de los hombres $(8,0 \%)$, tal como se muestra en la Tabla 5. No se observan diferencias significativas según lugar de trabajo.

El 2,3\% de los participantes refirió tener ideas suicidas, no existiendo diferencias significativas por género, ni por lugar de trabajo. Por lo tanto, 1 de cada 5 trabajadores que señaló su deseo de estar muerto $(12,2 \%)$, además, ha pensado en matarse $(2,3 \%)$. En los hombres esta razón fue mayor: 2 de cada 5 .

Dado que el deseo de estar muerto es un evento extremo y se relaciona con el riesgo suicida, se exploró su asociación con los otros indicadores de salud mental. Las personas que señalaron tener este deseo tenían un puntaje significativamente más alto en el GHQ-12 (22,6 $\pm 5,8$ vs $15,4 \pm 5,9$; $\mathrm{p}<0,001)$ y en el PHQ-9 $(15,1 \pm 5,9$ vs $7,7 \pm 5,0$; $\mathrm{p}<0,001)$. De igual forma, el $90,6 \%$ de quienes tenían el deseo de estar muertos son un caso positivo en el tamizaje del GHQ-12, en contraste con $54,5 \%$ entre quienes no tenían este deseo $(\mathrm{p}<0,001)$, indicando la asociación entre los indicadores negativos de salud mental y el riesgo suicida, entre estos trabajadores. 
Tabla 5. Indicadores de Salud Mental entre los trabajadores de salud que participaron en el estudio, según sexo y lugar de trabajo $(n=1.934)$

\begin{tabular}{|c|c|c|c|c|c|c|c|c|}
\hline \multirow[t]{2}{*}{ Variables } & \multirow{2}{*}{$\begin{array}{l}\text { Toda la } \\
\text { muestra }\end{array}$} & \multicolumn{2}{|c|}{ Género } & \multirow{2}{*}{$\begin{array}{c}\text { DM } \\
\mathbf{p} \\
\text { value }\end{array}$} & \multicolumn{3}{|c|}{ Tipo de Centro* } & \multirow{2}{*}{$\begin{array}{c}\text { DM } \\
\mathbf{p} \\
\text { value }\end{array}$} \\
\hline & & Mujer & Hombre & & Hospitales & CAA & Otros & \\
\hline $\begin{array}{l}\text { Puntaje total en el GHQ-12 } \\
\text { (promedio } \pm \mathrm{DE} \text { ) }\end{array}$ & $\begin{array}{c}16,3 \pm \\
6,3\end{array}$ & $\begin{array}{c}16,8 \pm \\
6,3\end{array}$ & $\begin{array}{c}14,9 \pm \\
6,3\end{array}$ & $<0,001$ & $16,3 \pm 6,4$ & $\begin{array}{c}16,3 \pm \\
6,3\end{array}$ & $\begin{array}{c}16,3 \pm \\
6,3\end{array}$ & 0,987 \\
\hline $\begin{array}{l}\text { Casos positivos en el screening } \\
\text { con GHQ-12 (> } 5 \text { puntos) (\%) }\end{array}$ & $58,9 \%$ & $61,9 \%$ & $50,3 \%$ & $<0,001$ & $58,7 \%$ & $58,4 \%$ & $63,3 \%$ & 0,582 \\
\hline \multirow{6}{*}{$\begin{array}{l}\text { Distribución porcentual de los } \\
\text { casos según intensidad del } \\
\text { cuadro depresivo (PHQ-9) } \\
\text { - Ausente o mínimo (0-4) } \\
\text { - Leve (5-9) } \\
\text { - Moderado (10-14) } \\
\text { - Moderado-grave (15-19) } \\
\text { - Grave (20-27) }\end{array}$} & & & & & & & & \\
\hline & $27,0 \%$ & $22,3 \%$ & $40,3 \%$ & $<0,001$ & $29,2 \%$ & $25,3 \%$ & $23,0 \%$ & 0,448 \\
\hline & $36,2 \%$ & $36,6 \%$ & $34,9 \%$ & $<0,001$ & $35,1 \%$ & $36,8 \%$ & $39,6 \%$ & 0,448 \\
\hline & $21,5 \%$ & $23,5 \%$ & $15,8 \%$ & & $20,4 \%$ & $22,7 \%$ & $20,9 \%$ & \\
\hline & $9,8 \%$ & $11,1 \%$ & $6,0 \%$ & & $10,4 \%$ & $8,9 \%$ & $10,8 \%$ & \\
\hline & $5,6 \%$ & $6,5 \%$ & $3,0 \%$ & & $4,9 \%$ & $6,3 \%$ & $5,8 \%$ & \\
\hline $\begin{array}{l}\text { Casos que señalan tener el deseo } \\
\text { de estar muertas/os (\%) }\end{array}$ & $12,2 \%$ & $13,6 \%$ & $8,0 \%$ & 0,001 & $11,8 \%$ & $12,8 \%$ & $10,8 \%$ & 0,729 \\
\hline $\begin{array}{l}\text { Casos que señalan tener ideas } \\
\text { de matarse }(\%)\end{array}$ & $2,3 \%$ & $2,0 \%$ & $3,2 \%$ & 0,110 & $2,4 \%$ & $1,9 \%$ & $3,6 \%$ & 0,431 \\
\hline $\begin{array}{l}\text { Participantes que sienten que } \\
\text { necesitan apoyo psicológico (\%) }\end{array}$ & $51,1 \%$ & $55,7 \%$ & $37,7 \%$ & $<0,001$ & $50,6 \%$ & $51,8 \%$ & $49,6 \%$ & 0,834 \\
\hline $\begin{array}{l}\text { Casos que han recibido apoyo } \\
\text { psicológico, entre quienes } \\
\text { sienten que la necesitan (\%) }\end{array}$ & $37,9 \%$ & $37,9 \%$ & $31,2 \%$ & 0,083 & $40,5 \%$ & $33,3 \%$ & $33,3 \%$ & 0,067 \\
\hline
\end{tabular}

Nota: $\mathrm{DE}=$ Desviación estándar, $\mathrm{DM}=$ Diferencia de medias. Hosp = Hospitales; $\mathrm{CAA}=$ Centros de atención ambulatoria.

\section{Salud mental y acceso a apoyo psicológico}

El 51,1\% de los encuestados sintió que ha necesitado apoyo psicológico durante este tiempo de pandemia. Esta cifra fue notoriamente más alta entre las mujeres y no hubo diferencias según lugar de trabajo (Tabla 5).

Quienes manifestaron la necesidad de recibir apoyo psicológico tenían peores indicadores de salud mental: en este grupo, los valores en el GHQ-12 y en el PHQ-9, fueron significativamente más elevados que quienes no lo manifestaron. De igual forma, dentro de este grupo, 77,7\% puntuaba como caso positivo en el tamizaje del GHQ-12, siendo solo 39,2\% en el otro grupo (datos no mostrados).

En la Tabla 5 se aprecia que solo un tercio de quienes señalaron necesitar ayuda psicológica la obtuvieron, siendo un poco mayor entre las mujeres y entre quienes trabajan en hospitales. Entre los que recibieron ayuda: $17,3 \%$ fue solo en forma presencial, $68,8 \%$ solo a distancia (llamada telefónica, videollamada, por chat, etc.) y 13,9\% recibió una combinación de ambas formas. Entre los que recibieron ayuda presencial, 53,1\% sentía que les ayudó "mucho" o "muchísimo", cifra que fue de $60,5 \%$ entre quienes recibieron apoyo a distancia (datos no mostrados).

Entre quienes refirieron el deseo de estar muerto, $82,1 \%$ señaló que había sentido la necesidad de apoyo psicológico. Solo un tercio de quienes deseaban estar muertos tuvieron alguna forma de apoyo psicológico, en su mayoría a distancia.

\section{Discusión}

Existe un alto porcentaje de trabajadores de la salud con síntomas de malestar psicológico, depresión e ideas suicidas, similar a lo reportado en la mayoría de los estudios realizados en este 
tiempo ${ }^{10,11}$ y bastante más alto que las cifras de prevalencia reportadas previo a la pandemia en nuestro país ${ }^{25,28}$. A la vez, es preocupante que solo un tercio de los trabajadores que sienten que necesitan ayuda psicológica la han recibido, lo que nos señala necesidades de atención no satisfechas con las medidas que se habían tomado hasta el momento de la encuesta ${ }^{29}$, lo cual requiere una pronta acción. En especial, destacamos $2 / 3$ de los trabajadores de la salud que señalan haber tenido deseos de estar muertos, no han recibido apoyo, lo que es una situación muy preocupante.

Otro aspecto relevante es la mayor frecuencia de problemas de salud mental entre las trabajadoras. Si bien, en todos los estudios de prevalencia de trastornos mentales -incluidos los trabajadores sanitarios $^{30}$-, las mujeres tienen cifras más elevadas que los hombres, cuya explicación puede analizarse mejor desde una perspectiva de género que en aspectos puramente biológicos ${ }^{31}$. Nuestro estudio muestra que ellas reportan con mayor frecuencia varias condiciones de riesgo, incluyendo más experiencias de ser objeto de estigmatización y violencia, así como un menor apoyo recibido para quienes lo necesitan. Una gran proporción de los profesionales de la salud son mujeres que enfrentan un mayor riesgo de infección, morbilidad y muerte por su profesión, y al mismo tiempo, actúan con más frecuencia como las principales cuidadoras dentro del hogar. Estas cargas “extra”, como se ha visto en epidemias previas ${ }^{32,33}$, ponen a las trabajadoras de la salud en mayor riesgo de desarrollar patología psiquiátrica. Al mismo tiempo, los hombres solicitan menos ayuda y servicios de salud mental cuando los necesitan, al menos en población general ${ }^{34}$.

Un hallazgo destacable, que hasta la fecha no ha sido reportado, es la mayor frecuencia de condiciones de riesgo en los trabajadores de centros de atención ambulatoria (atención primaria y especialidades). En general, la mayoría de los países han puesto mayor preocupación en los equipos que laboran en áreas de alta especialización hospitalaria (como unidades de pacientes críticos) y en nuestro estudio se aprecia que los trabajadores de hospitales con necesidades de apoyo psicológico acceden a él con mayor frecuencia que sus contrapartes en atención ambulatoria. Esto es importante para orientar más recursos hacia los centros de atención ambulatoria y facilitar su acceso a servicios o intervenciones de apoyo; y, además, para potenciar el desarrollo de estrategias de tipo local, más cercana al lugar directo de trabajo (servicios clínicos, CESFAM, etc.) y que han demostrado ser efectivas, tal como fue señalado en una revisión hecha por miembros de nuestro equipo ${ }^{17}$.

Nuestro estudio presenta algunas limitaciones que se explican por el contexto donde fue hecho y que deben tenerse en cuenta para interpretar los resultados en forma correcta. La primera de ellas es la baja tasa de participación, de 3,4\% en la primera fase de la encuesta, y luego la imposibilidad de calcular esta tasa durante la segunda fase. No se puede asegurar la representatividad de la muestra respecto del universo de nuestros trabajadores de la salud. Sin embargo, la mayoría de los trabajos que utilizan estas metodologías tienen tasas bajas $\mathrm{s}^{35,36} \mathrm{o}$ ni siquiera son reportadas ${ }^{11,20}$. Esta limitación conlleva un potencial sesgo de selección que pudiera sobredimensionar las prevalencias observadas, aunque no tenemos ninguna evidencia empírica que esto haya sido así.

Otra limitación es que el uso de encuestas on-line hace menos probable la participación de personal de más edad (con menor alfabetización digital) o con menos recursos de acceso a tecnología ${ }^{37}$. Para reducir esta limitación, se hizo un esfuerzo especial en los centros que participaron, para que nuestra muestra contenga una diversidad de trabajadores y de rangos de edad, lo que contrasta con otros estudios que se focalizaron solo en profesionales ${ }^{10,11,20}$.

A pesar de estas limitaciones, nuestro estudio logró tener una muestra mayor a la estimada para un estudio de este tipo, con una diversidad de género, edad, tipo y lugar de trabajo, que parece puede ser un buen reflejo de la totalidad de los trabajadores sanitarios de nuestro país. Además, el modelo del problema que guió la construcción de nuestra encuesta incorpora todas variables que han sido asociadas con el riesgo de presentar un problema de salud mental en revisiones recientes ${ }^{15}$, lo que permite cumplir con nuestro propósito principal: aportar al análisis y desarrollo de las medidas, intervenciones y políticas que contribuyan a proteger la salud mental de los trabajadores sanitarios.

Los hallazgos aquí presentados confirman la urgencia de tomar medidas integrales que permitan abordar la salud mental del personal sanitario, tanto en la prevención como en la detección y 
tratamiento, relevando la necesidad de un enfoque de género y un mayor énfasis en el cuidado de los equipos de atención primaria y de otros centros de atención ambulatoria. Igualmente, constituyen el punto inicial para un estudio de cohorte en curso que permitirá caracterizar el desarrollo del problema, en el contexto de una pandemia en plena evolución.

\section{Referencias}

1. Organización Mundial de la Salud (OMS). Weekly Operational Update on COVID-19. 5 January 2021 [Internet]. 2021 [citado 7 de enero de 2021]. Disponible en: https://www.who.int/publications/m/item/ weekly-epidemiological-update---5-january-2021.

2. Ministerio de Salud Chile. Cifras Oficiales [Internet]. 2021 [citado el 7 de enero de 2021]. Disponible en: https://www.gob.cl/coronavirus/cifrasoficiales/

3. Petersen E, Koopmans M, Go U, Hamer DH, Petrosillo $\mathrm{N}$, Castelli F, et al. Comparing SARS-CoV-2 with SARS$\mathrm{CoV}$ and influenza pandemics. Lancet Infect Dis. 2020; 20: e238-44.

4. Stawicki SP, Jeanmonod R, Miller AC, others. The 2019-2020 Novel Coronavirus (Severe Acute Respiratory Syndrome Coronavirus 2) Pandemic: A Joint American College of Academic International Medicine-World Academic Council of Emergency Medicine Multidisciplinary COVID-19 Working Group Consensus Paper. J Glob Infect Dis. 2020; 12 (2): 47-93.

5. Organizacion de Naciones Unidas (ONU). Policy brief: COVID-19 and the Need for Action on Mental Health. ONU. 2020 may.

6. Esterwood E, Saeed S. Past epidemics, natural disasters, COVID19, and mental health: learning from history as we deal with the present and prepare for the future. Psychiatr Q. 2019; 91: 1121-33.

7. Burrone M, Reginatto G, Solís-Soto M, Basagoitia A, Irarrázaval M, Rincón $\mathrm{P}$, et al. Coronavirus e impacto psicosocial en Chile. Carta al editor. Rev Med Chile 2020; 148: 1224-6.

8. Centro UC, Asociación Chilena de Seguridad. Encuestas y estudios longitudinales. Termómetro de la Salud Mental en Chile 2020.

9. Greenberg N, Docherty M, Gnanapragasam, S Wessely S. Managing mental health challenges faced by healthcare workers during COVID-19 pandemic. BMJ. 2020; 368.

10. Hossain MM, Tasnim S, Sultana A, others. Epidemiology of mental health problems in COVID-19: a review [version 1; peer review: 2 approved]. F1000Research. 2020; 9: 636.

11. Lai J, Ma S, Wang Y, Cai Z, Hu J, Wei N, et al. Factors Associated With Mental Health Outcomes Among Health Care Workers Exposed to Coronavirus Disease 2019. JAMA Netw open. 2020; 3 (3): e203976.

12. Huang L, Xu F, Liu H. Emotional responses and coping strategies of nurses and nursing college students during COVID-19 outbreak. medRxiv. 8 de marzo de 2020;2020.03.05.20031898.

13. Brooks SK, Dunn R, Amlôt R, Rubin GJ, Greenberg N. A Systematic, Thematic Review of Social and Occupational Factors Associated With Psychological Outcomes in Healthcare Employees During an Infectious Disease Outbreak. J Occup Environ Med. 2018; 60 (3): 248-57.

14. Cabello IR, Echavez JFM, Serrano-Ripoll MJ, Fraile-Navarro D, Roque MAF de, Moreno GP, et al. Impact of viral epidemic outbreaks on mental health of healthcare workers: a rapid systematic review. medRxiv. 2020. 2020.04.02.20048892.

15. De Brier N, Al E. Factors Affecting Mental Health of Health Care Workers During Coronavirus Disease Outbreaks: A Rapid Systematic Review. PsyArXiv. 2020 abr.

16. Organización Mundial de la Salud (OMS). Garantizar la seguridad de los trabajadores de la salud para preservar la de los pacientes [Internet]. Comunicado de prensa. 2020 [citado 7 de enero de 2021]. Disponible en: https:// www.who.int/es/news/item/17-09-2020-keep-healthworkers-safe-to-keep-patients-safe-who

17. Traub C, Sapaj J. Personal Sanitario y Pandemia COVID-19 en Chile: Desafíos en Salud Mental. Rev Med Chile 2020; 148: 1197-204.

18. Papoutsi E, Giannakoulis V, Ntella V, Pappa S, Katsaounou P. Global burden of COVID-19 pandemic on healthcare workers. ERJ Open Res. 2020; 6 (2): 00195 2020.

19. Sepúlveda S, Miranda N. Actas del Minsal: 6.840 funcionarios de la salud se han contagiado y más de 10 mil han partido a cuarentena preventiva [Internet]. CIPER Chile. 2020 [citado 7 de enero de 2021]. Disponible en: https://www.ciperchile.cl/2020/05/30/actas-del-minsal6-840-funcionarios-de-la-salud-se-han-contagiado-ymas-de-10-mil-han-partido-a-cuarentena-preventiva/

20. Urzúa A, Samaniego A, Caqueo-Urízar A, Zapata A, Irarrázaval M. Salud mental en trabajadores de la salud durante la pandemia por COVID-19 en Chile. Rev Med Chile 2020; 148: 1121-7.

21. Goldberg D, Williams PA. User's Guide to the General Health Questionnaire. $1^{\text {a }}$ ed. Oxford, England: NERF-NELSON 1988; 129.

22. Araya R, Wynn R, Lewis G. Comparison of two self 
administered psychiatric questionnaires (GHQ-12 and SRQ-20) in primary care in Chile. Soc Psychiatry Psychiatr Epidemiol. 1992; 27: 168-73.

23. Garmendia ML. Análisis factorial: una aplicación en el cuestionario de salud general de Goldberg, versión de 12 preguntas. Rev Chil salud pública. 2007; 11 (2): 57-65.

24. Kroenke K, Spitzer R, Williams J. The PHQ-9. Validity of a Brief Depression Severity Measure. J Gen Intern Med. 2001; 16: 606-13.

25. Saldivia S, Aslan J, Cova F, Vicente B, Inostroza C, Rincón P. Propiedades psicométricas del PHQ-9 (Patient Health Questionnaire) en centros de atención primaria de Chile. Rev Med Chile 2019; 147: 53-60.

26. Al-Halabí S, Sáiz P, Burón P, Garrido M, Benabarre A, Jiménez E, et al. Validación de la versión en espanñol de la Columbia-Suicide Severity Rating Scale (Escala Columbia para Evaluar el Riesgo de Suicidio). Rev Psiquiatr Salud Ment. 2016; 9 (3): 134-42.

27. Harris PA, Taylor R, Thielke R, Payne J, Gonzalez N, Conde JG. Research electronic data capture (REDCap)--A metadata-driven methodology and workflow process for providing translational research informatics support. En: J Biomed Informa. 2009; 377-81.

28. Vicente B, Khon R, Rioseco P, Saldivia S, Baker C, Torres $\mathrm{S}$. Population prevalence of psychiatric disorders in Chile: 6-month and 1-month rates. Br J Psychiatry. 2004; 184: 299-305.

29. Ministerio de Salud Chile. Programa Saludablemente [Internet]. 2020 [citado 7 de enero de 2021]. Disponible en: https://www.minsal.cl/saludablemente/

30. Guille C, Frank E, Zhao Z, others. Work-Family Conflict and the Sex Difference in Depression Among Training
Physicians. JAMA Intern Med. 2017; 177 (12): 1766-72.

31. Johnson JL, Greaves L, Repta R. Better science with sex and gender: Facilitating the use of a sex and gender-based analysis in health research. Int J Equity Health. 2009; 8 (1): 1-11.

32. Chong M-Y, Wang W-C, Hsieh W-C, Lee C-Y, Chiu $\mathrm{N}-\mathrm{M}$, Yeh W-C, et al. Psychological impact of severe acute respiratory syndrome on health workers in a tertiary hospital. Br J Psychiatry. 2004; 185: 2.

33. Tam CWC, Pang EPF, Lam LCW, Chiu HFK. Severe acute respiratory syndrome (SARS) in Hong Kong in 2003: stress and psychological impact among frontline healthcare workers. Psychol Med. 2004; 34 (7): 1197 204.

34. Sagar-Ouriaghli I, Godfrey E, Bridge L, Meade L, Brown JS. Improving mental health service utilization among men: A systematic review and synthesis of behavior change techniques within interventions targeting help-seeking. Am J Mens Health. 2019; 13 (3): 1557988319857009.

35. Kongsved SM, Basnov M, Holm-Christensen K, Hjollund NH. Response rate and completeness of questionnaires: a randomized study of Internet versus paper-and-pencil versions. J Med Internet Res. 2007; 9 (3): e25.

36. Ebert JF, Huibers L, Christensen B, Christensen MB. Paper- or web-based questionnaire invitations as a method for data collection: cross-sectional comparative study of differences in response rate, completeness of data, and financial cost. J Med Internet Res. 2018; 20 (1): e24.

37. Millán M, Finkel L. Encuestas por Internet y nuevos procedimientos muestrales. Panor Soc. 2019; 30: 41-53. 